\title{
AVALIAÇÃO DOS ÍNDICES DE MORTALIDADE POR SUICÍDIO NO MUNICÍPIO DE SOUSA-PB NOS ANOS DE 2016 E 2017
}

\section{EVALUATION OF SUICIDE MORTALITY INDICES IN SOUSA-PB CITY IN 2016 AND 2017}

\author{
Sabrina Kénia Alves Celeste ${ }^{1}$ \\ Maximiliano Pucci Andrade de Oliveira ${ }^{2}$ \\ Renata Livia Silva Fonsêca Moreira de Medeiros ${ }^{3}$ \\ Macerlane de Lira Silva ${ }^{4}$
}

RESUMO: Introdução: A palavra suicídio tem origem no latim e significa sui = si mesmo e caedes = ação de matar, isto é, a morte de si mesmo. O suicídio é definido como uma morte por lesão autoprovocada, um ato voluntário contra a própria vida que resulta em morte, caracterizando uma trágica e intempestiva perda de vida humana. A crise suicida é determinada por comportamentos potencialmente auto lesivos com evidência de que a pessoa tem o pensamento de se matar, sendo que o resultado de um ato suicida pode variar desde a não ocorrência de lesão até a morte. Objetivo: O presente trabalho tem como objetivo analisar, através das variáveis demográficas, o índice de mortes por suicídio no município de Sousa, interior da Paraíba, no período de 2016 e 2017. Metodologia: Trata-se de um estudo epidemiológico de carácter descritivo, retrospectivo com abordagem quantitativa. A pesquisa foi baseada em dados secundários coletados no Sistema de Informação de Mortalidade (SIM), com objetivo de analisar, através das variáveis demográficas, o índice de mortes por suicídio no Município de Sousa-PB, interior da Paraíba no período de 2016/2017. Resultados e Discussões: Segundo os resultados da pesquisa os homens cometem mais suicídios que as mulheres.

\footnotetext{
${ }^{1}$ Discente do curso de medicina da Faculdade Santa Maria.

$2{ }^{2}$ Docente da Faculdade Santa Maria - FSM. Médico Psiquiatra formado pela Universidade de Alfenas-MG, Residência em Clínica Médica na Casa de Saúde Santa Marcelina- SP e Residência em Psiquiatria no FHEMIG Hospital Raul Soares- MG.

${ }^{3}$ Docente da Faculdade Santa Maria - FSM. Enfermeira pela Universidade Federal da Paraiba-UFPB. Especialista em Saúde Pública pela FACISA. Mestre em Enfermagem pela Universidade Federal da Paraíba -UFPB. Doutora em Pesquisa em Cirurgia pela Faculdade de Ciências Médicas Santa Casa de São Paulo - FCMSP.

${ }^{4}$ Docente da Faculdade Santa Maria - FSM. Graduação em Enfermagem pela FSM Cajazeiras, especialização em política e gestão do cuidado com ênfase no apoio matricial pela UFPB e mestrado em Saúde Coletiva pela UNISANTOS.
} 
Estima-se que o suicídio seja a terceira causa de morte na faixa etária dos 15-34 anos. Quanto a população idosa, os dados mostram também um índice relevante entre essa população. A pesquisa chama atenção para o local de maior índice de ocorrência de suicídios que ocorrem nos próprios ambientes domiciliares e como método usado na execução do ato suicida destaca-se mortes por enforcamento/estrangulamento/sufocamento como principal meio prevalente. Conclusão: O estudo mostra que, independente da faixa etária, sexo, idade, devese ter sempre um olhar holístico para atitudes e comportamentos das pessoas que muitas vezes, de maneira informal, acenam por socorro, mas diversas vezes são ignoradas, negligenciadas e/ou não são observadas como deveriam perante os familiares, amigos, companheiro, sociedade e autoridades.

Descritores: Mortalidade. Suicídio. Variáveis demográficas.

ABSTRACT: Introduction: The word suicide originates from Latin and means sui = self and caedes = killing action, that is, the death of oneself. Suicide is defined as a death by self-harm, a voluntary act against one's life that results in death characterizing a tragic and untimely loss of human life. The suicidal crisis is defined as potentially self-injurious behaviors with evidence that the person has the thought of killing himself, and the result of a suicidal act can vary from no injury to death. Objective: This study aims to analyze, through demographic variables, the rate of suicide deaths in the municipality of Sousa, interior of Paraiba, in the period 2016 and 2017. Methodology: This is a descriptive epidemiological method. retrospective with quantitative approach. The research was based on secondary data collected in the Mortality Information System (SIM), aiming to analyze, through demographic variables, the suicide death rate in the city of Sousa-PB, Paraíba, Brazil, in 2016/2017. Results and Discussion: According to the survey results men commit more suicides than women. Suicide is estimated to be the third leading cause of death in the 15-34 age group. Regarding the elderly population, the data also show a relevant index among this population. The research draws attention to the place of highest occurrence of suicides that are in their own home environments and as a method used in the execution of suicide act hangs / strangulation / suffocation deaths as the main prevalent means. Conclusion: The study shows that regardless of age, gender, age, one should have a holistic look at the attitudes and behaviors of people who often informally wave for help but are often ignored, neglected and / or not observed. as they should to family, friends, partner, society and authorities.

Descriptors: mortality, suicide, demographic variables. 


\section{INTRODUÇÃO}

A palavra suicídio tem origem no latim e significa $s u i=$ si mesmo e caedes $=$ ação de matar, isto é, a morte de si mesmo. O suicídio é definido como uma morte por lesão autoprovocada, um ato voluntário contra a própria vida que resulta em morte, caracterizando uma trágica e intempestiva perda de vida humana. A crise suicida define-se como comportamentos potencialmente autolesivos com evidência de que a pessoa tem o pensamento de se matar, sendo que o resultado de um ato suicida pode variar desde a não ocorrência de lesão até a morte (WERLANG; BORGES; FENSTERSEIFER, 2005).

O suicídio está ligado as necessidades frustradas, insatisfação, desamparo, falta de esperança, luto, violência, ocorrendo um estreitamento do seu mundo interior de modo que o sujeito sente a necessidade de fuga para determinados problemas que não conseguem resolver e que estão causando demasiado sofrimento. Existem alguns indicadores de risco que podem ser detectados, tais como: depressão, melancolia, tristeza, desesperança, ansiedade, grande impulsividade e agressividade, abuso de álcool e drogas, dificuldades nos relacionamentos, isolamento social, luto pela perda de entes queridos, história de suicídios na família, entre outros (SADOCK; SADOCK, 2007).

Assim, entende-se que o suicídio resulta de uma complexa interação de fatores (biológicos, genéticos, psicológicos, sociológicos, culturais e ambientais), de modo que o desafio chave consiste na prevenção e identificação de pessoas que são vulneráveis. Desse modo, saber identificar e compreender as circunstâncias que induzem ao comportamento suicida para poder estruturar intervenções eficazes e evitá-lo. No entanto, uma avaliação abrangente dos comportamentos suicidas é fundamental para uma intervenção terapêutica eficaz, onde o objetivo da avaliação é ofertar informação quanto a prevenção e aconselhamento (OMS, 2006).

Uma tentativa de suicídio prévia é o principal fator de risco para uma segunda tentativa. Após a primeira tentativa, estima-se que o risco de suicídio aumente em, 
pelo menos, cem vezes em comparação aos índices presentes na população em geral (OWENS; HORROCKS; HOUSE, 2002).

Refletir sobre o suicídio é também investigar por que este acontecimento tem sido silenciado durante muito tempo pela nossa sociedade, autoridades, responsáveis, profissionais da saúde e familiares, camuflando um grave problema de saúde pública no Brasil e no mundo (BOTEGA, 2002). Assim, é relevante avaliar o risco de suicídio, reconhecendo os aspectos modificáveis e tratáveis, como também maneiras de proteção que orientem o tratamento do paciente e a necessidade de controle (HALES; YUDOFSKY; GABBARD; 2012).

Segundo a Fundação Oswaldo Cruz (FIOCCRUZ) (2014), a maior parte das pessoas que pensam em praticar o suicídio enfrenta uma doença mental que altera, de maneira radical, a percepção da realidade, interferindo no julgamento e tomada de decisões, sendo o tratamento da doença é a melhor maneira de prevenção (VARELA, 2016).

Segundo a Organização Mundial de Saúde (2014), o suicídio é um acontecimento crescente em diversos países. Mais de 800 mil pessoas suicidam-se todos os anos e esse número deve chegar a 1,6 milhão de mortes em 2020. Contudo, a OMS acredita que esse número esteja subestimado em vinte vezes devido à subnotificação ou inexistência de registros de ocorrências.

A cada quarenta e cinco segundos ocorre um suicídio em algum lugar do mundo. Existe um contingente de 1920 pessoas que tiram a própria vida diariamente. Atualmente, essa cifra supera, ao final de um ano, a soma de todas as mortes causadas por homicídios, acidentes de trânsito e conflitos civis (VARNIK, 2012; WHO, 2014).

Considerando a gravidade do problema, é relevante o desenvolvimento de ações, serviços e programas de saúde direcionados para a população de risco, visando a prevenção do suicídio (LOVISI et al., 2009).

Compreende-se que o tema se torna relevante uma vez que o indivíduo demonstra sinais de que precisa de ajuda e, em sua maioria, é negligenciado. Dessa forma, por se tratar de um sério problema de saúde pública, existir pouca discussão sobre o assunto e ainda ser tratado como "tabu", surgiu o interesse de avaliar os índices de mortes por suicídio no município de Sousa, Paraíba. Desse modo, o 
presente trabalho objetiva analisar, através das variáveis demográficas, o índice de tais eventos na localidade descrita.

\section{MATERIAIS E MÉTODOS}

Trata-se de um estudo epidemiológico de carácter descritivo, retrospectivo, com abordagem quantitativa dos dados.

A pesquisa foi realizada no município de Sousa-PB, localizado no interior da Paraíba, apresenta uma área territorial de 738,547 km2 e uma população de 69.196 habitantes, sendo o sexto mais populoso do estado, o primeiro de sua microrregião e o segundo da mesorregião, conforme dados do Instituto Brasileiro de Geografia e Estatística (IBGE) (BRASIL, 2016).

A população do estudo foi definida a partir da faixa etária acima de 10 anos de idade. A pesquisa foi baseada em dados secundários coletados no SIM, de modo que a operacionalização da coleta de dados ocorreu mediante as informações contidas no banco de dados disponível ao público de forma online, assim, por ser de domínio público, a pesquisa dispensa o Termo de Consentimento Livre e Esclarecido (TCLE).

Inicialmente foi realizada uma busca no SIM a fim de obter, através das variáveis demográficas, os índices registrados estatisticamente do número de óbitos por suicídio nos anos de 2016/2017 na cidade de SOUSA-PB. Esses foram analisados por meio de estatística descritiva definida como variáveis: sexo, idade, estado civil, local de ocorrência do evento e meio utilizado para prática do ato. Para caracterização dos óbitos foram utilizados os dados como causa básica do suicídio codificada na Classificação Internacional de Doenças (CID), o qual compreende as categorias X-60 a X-84: lesão autoprovocada voluntariamente.

Os dados foram analisados estatisticamente mediante utilização do programa Statistical Pakageforthe Social Sciens (SPSS) versão 21.0, e averiguados conforme literatura, com auxílio de estatística epidemiológica descritiva, retrospectiva com 
abordagem quantitativa indicada para estudos nos quais se pretende quantificar e expor os resultados sem modificá-los.

Todas as etapas de investigação seguiram as diretrizes da resolução 466/12 do CNS, que regulamenta a pesquisa realizada de forma individual ou coletiva envolvendo seres humanos de forma direta ou indireta (BRASIL, 2012).

\section{RESULTADOS E DISCUSSÃO}

Segundo Cataldo Neto; Gauer; Futado, et al. (2003), os homens cometem mais suicídio que as mulheres. O sexo feminino tenta com maior frequência, porém, a maior prevalência de êxito letal ocorre com a população masculina.

TABELA 01 - Dados obtidos no Sistema de Informação de Mortalidade (SIM).

\begin{tabular}{lcc}
\hline SEXO & $\mathbf{2 0 1 6}$ & $\mathbf{2 0 1 7}$ \\
Masculino & 06 & 07 \\
Feminino & 01 & 00 \\
Idade & 00 & 01 \\
$15-19$ anos & 02 & 01 \\
$20-29$ anos & 01 & 04 \\
$30-39$ anos & 01 & 00 \\
$60-69$ anos & 02 & 01 \\
$70-79$ anos & 01 & 00 \\
$80 a n o s$ ou mais & & \\
Estado civil & 02 & 03 \\
Solteiro & 01 & 01 \\
Casado & 03 & 02 \\
Ignorado & 01 & 01 \\
Outros & & \\
Local de ocorrência do evento & 05 & 05 \\
Domicílio & 01 & 00 \\
Via pública & 00 & 01 \\
Hospital & 01 & 01 \\
Outros & & \\
Meio utilizado para prática evento suicida & 06 & 05 \\
Enforcamento/estrangulamento/sufocamento & 01 & 00 \\
Envenenamento & 00 & 02 \\
Arma de fogo & & \\
\hline TOTAL & &
\end{tabular}

Fonte: SIM (2019). 
Os registros quanto a idade mostram que o suicídio é um fato que vem aumentando e que engloba uma faixa etária diversa desde adolescentes, adulto jovem e idosos com 80 anos ou mais, conforme dados obtidos no SIM do município de Sousa-PB, correspondente aos anos 2016 e 2017, o que mostra a importância de uma visão mais atenta para as diversas mudanças de comportamento, independente da idade.

De acordo com Rothes (2006) e Vidal; Gontijo; Lima (2013), estima-se que o suicídio seja a terceira causa de morte na faixa etária dos 15-34 anos. Quanto à população idosa os dados mostram um índice relevante entre essa população. Os idosos tentam menos suicídio que os mais jovens, embora, quando tentam, o método é altamente letal, como: enforcamento, precipitações (queda de altura), veneno e armas de fogo. O idoso geralmente busca circunstâncias para não serem descobertos, nem salvo (BARRERO, MATUSEVICH, 2009).

Idosos deprimidos com dificuldades de lidarem com rejeição ou críticas e que apresentam hostilidade em seus relacionamentos tendem a sentir falta de apoio social, tornando-se mais propensos a considerarem ou tentarem o suicídio. Assim, os idosos que apresentam uma história de dificuldades interpessoais devem ser cuidadosamente monitorados para comportamento suicida (HARRISON et al, 2010).

Idosos deprimidos enquadram-se na "Síndrome do Ninho Vazio" quando, permanecendo sozinhos, sentem-se abandonados, entrando em questionamentos e pensamentos que podem levar ao desencadeamento de ideias suicidas.

Quando analisados os índices de mortes por suicídio quanto ao estado civil pode-se constatar que o índice é maior entre solteiros quando comparado com os casados, retratando, assim, maior risco naqueles sem vínculo estável, permanecendo esses mais vulneráveis. A pesquisa também chama atenção para os casos ignorados que obtiveram uma alta prevalência.

A pesquisa chama atenção para o local de maior índice de ocorrência de suicídios, os próprios ambientes domiciliares, mostrando a importância do reconhecimento prévio das alterações comportamentais, atitudes suspeitas e/ou alguma doença psíquica para que seja possível intervir de forma precoce. Outro ponto relevante é o meio utilizado para execução do ato suicida, com mortes por 
enforcamento/estrangulamento/sufocamento como principal meio usado, demonstrando que os suicidas agem através de planejamento para executarem o ato de forma letal e sem erro; armas de fogo e envenenamento ocorrem em menor proporção.

Estudos revelam que três características em particular são próprias do estado das mentes suicidas: ambivalência, impulsividade e rigidez. A maioria das pessoas já teve pensamentos e sentimentos confusos quanto ideação e prática suicida, vivenciados pelos momentos de tristeza, angústia e desespero, por situações vividas em que o desejo de viver e morrer batalham em uma gangorra nesses indivíduos, vivendo situações em que a única saída que encontram para alívio da dor seria a morte, o suicídio, não por desejarem a morte, mas sim pelo desejo do alívio do sofrimento, vivendo, dessa maneira, a ambivalência entre o viver e o morrer (OMS, 2000).

Outra característica vivida pelo suicida é a impulsividade, sendo o impulso do indivíduo suicida transitório, com duração de minutos ou horas, desencadeado por momentos negativos vividos e que no momento de angústia e sofrimento atua como um fator forte para cometer o suicídio devido à somatização dos eventos negativos desencadeados pelo dia-dia. Porém, o que queremos retratar na pesquisa é que possamos reconhecer em tempo hábil aquele momento de dor, angústia e sofrimento para ganhar tempo e mostrar outra visão da vida, ajudando a diminuir o desejo suicida (OMS, 2000).

No tocante ao momento de rigidez permanece o pensamento rígido $\mathrm{e}$ drasticamente de suicidar-se, não sendo capaz de perceber outra saída para solução dos problemas, fixando-se rigidamente no desejo de morrer. Leonel (2014) menciona que "tentativas de suicídio deveriam ser encaradas com seriedade, como um sinal de alerta, revelando a atuação de fenômenos psicossociais complexos".

A maioria das pessoas com pensamentos suicidas frequentemente dão sinais e fazem comentários sobre o desejo de morrer, que não aguentam mais aquela situação, acenando sinais de que se o fizer não fará falta e será alívio para as pessoas, e assim por diante. Todos esses pedidos de ajuda não podem ser ignorados. Estão presentes sentimentos como: tristeza, depressão, solidão, desamparo, desesperança, autodesvalorização. 
A maioria dos suicidas não consegue decidir-se entre viver ou morrer, essas pessoas jogam com a morte, deixando aos outros a missão de salvá-las. Difícil alguém cometer suicídio sem dizer a outra pessoa como está sentindo-se, sendo esse o momento de pedido de socorro, que na maioria das vezes é banalizado e até criticado (LOPES, 2007).

Apesar de o suicida ser infeliz, ansioso e preocupado, nem toda pessoa que se suicida pode ser diagnosticada como mentalmente enferma. É notável que um suicida está sob forte pressão e, em certo momento, enfrenta conflitos e desajustes mental. Todavia, uma pessoa pode tirar a sua vida estando lúcida e consciente. Mesmo aqueles que construíram um passado de glória e têm um presente promissor e de sucesso podem ter olhos completamente embaçados para ver o futuro (LOPES, 2007).

Uma abordagem calma e sem julgamentos é fundamental para facilitar a comunicação entre a pessoa com ideação suicida, dando-a atenção para ouvir com cordialidade, respeito e, principalmente, repassar segurança de que o seu desabafo permanecerá em sigilo e que pode contar com sua ajuda, respeito e atenção, podendo confiar no que for preciso, que está lá para apoiá-la.

Falar sobre o suicídio é de suma importância, uma vez que os fenômenos de doenças como a depressão e ideação suicida estão se tornando cada vez mais evidentes perante a sociedade e são caracterizados como problema de saúde pública, relevância, não somente de retratar sobre ideação suicida, mais também a prática e concretização do ato.

É preciso reconhecer quando determinada pessoa precisa de ajuda, e a partir de então entender qual o problema que está angustiando e causando demasiado sofrimento, que ela não consegue enxergar outra saída a não ser a morte. A prática do ato suicida acontece, não porque a pessoa deseja a morte, mas sim devido ao seu grau de sofrimento ser tão grande que ela quer matar a dor que está sentindo, e não morrer.

O acesso ao método para cometer suicídio é um fator determinante para indivíduo vir a cometer o suicídio ou desistir. Tentar reduzir o acesso a métodos de risco de cometer suicídio é uma estratégia efetiva de prevenção. 
Para ajudar faz-se necessário uma visão holística do assunto, observar com mais atenção os sinais e comportamentos das pessoas para entender as situações pela quais estão passando e procurar ajudá-las sem censurá-las, pois estas pessoas estão vivenciando um grau de tristeza tão profundo que não conseguem enxergar uma luz à sua frente que console a dor que assola a alma, seja essa dor desencadeada por diversos motivos, como: fim de relacionamentos, luto, causas de vergonha, atritos de identidade, solidão, frustações, problemas familiares, doenças mentais como ansiedade, depressão, esquizofrenia, dentre inúmeros outros fatores que acometem.

O suicídio é um ato irremediável que sempre produz mais dor que alívio. É preciso explanar mais sobre o assunto, pois, muitas vezes, as pessoas precisam de ajuda, pedem socorro, mas na maioria das vezes são ignoradas, tratadas como "fracas", "frescuras", sendo negligenciadas pela família, amigos, companheiro, sociedade, e até mesmo pelos profissionais da saúde.

Acontece, muitas vezes, até por falta de conhecimento quanto ao assunto, sendo importante e necessário falar sobre o suicídio, visto, ainda, que muitas vezes as pessoas falam pelo desejo de morrer, porém, são ignoradas pelo mito de que quem fala não faz, sendo um grande engano pensar que aquelas pessoas que falam no desejo ou ameaçam suicidar-se nunca tentarão. Pelo contrário, essas ameaças devem ser levadas à sério, não devendo subestimar os sinais emitidos por quem fala em suicídio.

$\mathrm{Na}$ maioria das vezes a pessoa que se suicida planeja como terminar sua existência dando indícios de sua intenção, porém, são negligenciadas. Quem já tentou, vai tentar de novo. Aqueles que flertam com a morte acabam dando as mãos a ela em uma viagem sem volta, sendo as diversas tentativas prévias o fator forte nessa busca pela morte.

Natrielli Filho (2005, p. 260) relata que "aproximadamente metade dos psiquiatras e $20 \%$ dos psicólogos perdem algum paciente em tratamento por suicídio", advertindo para importância do assunto. Observa-se, através dos estudos e pesquisas, que o suicídio está atingindo uma faixa etária diversificada, porém, com uma prevalência maior entre adolescentes, adultos jovens e idosos. A partir das estatísticas deve-se trabalhar com o desenvolvimento de campanhas educativas, 
retratando a relevância do tema, da importância do alerta e do reconhecimento precoce quando a pessoa dá indícios de sinais de sofrimentos e que está precisando de ajuda naquele momento de dor imensa vivida, quando não visualiza uma saída plausível para solução do problema. Falar da importância de uma palavra amiga naquele momento de angústia, do apoio familiar e da busca de ajuda profissional são pontos que, em determinado momento de tristeza, fará a diferença durante aquele cenário de sofrimento.

Segundo Lopes (2007), o suicídio é o ato voluntário e intencional de matar a si mesmo. É o assassinato de si mesmo. É o último e irreversível estágio da autodestruição. É a violência fatal contra si para pôr fim a uma dor maior do que a vontade de viver.

Mostrar para a pessoa que, por pior que seja aquele momento de dor e de questionamentos negativos, que algo aconteceu e não era para ter acontecido e que trouxe muito sofrimento, sempre existe uma saída e essa não é a morte, não é o suicídio. Mostrar para a pessoa que ela é importante e que existem outros meios para solução daquela situação vivenciada de dor e mostrar que o desabafo traz cura, alívio.

Gary Stuart alerta: "Um desejo suicida é um pedido de socorro. Se você ouvir esse grito, responda; se o grito for seu, procure ajuda".

\section{CONSIDERAÇÕES FINAIS}

Os dados apresentados nesse estudo indicam que a mortalidade por suicídio no município de Sousa-PB, referente aos anos 2016-2017, e mostram uma tendência diversificada das mortes por suicídio, mostrando que, a prevalência maior de óbitos por suicídio ocorre entre o sexo masculino, porém, com uma faixa etária diversificada, acometendo indivíduos adolescentes, adultos jovem e idosos com 80 anos ou mais, como mostram os resultados.

O estudo mostra que, independente da faixa etária, sexo, idade, se deve possuir um olhar holístico para atitudes e comportamentos das pessoas que, muitas 
vezes de maneira informal, acenam por socorro, mas diversas vezes são ignoradas, negligenciadas e/ou nem observadas como deveriam perante os familiares, amigos, companheiro, sociedade e autoridades.

Quanto aos profissionais da saúde a pesquisa tenta retratar a importância do olhar cuidadoso com o outro, da importância de uma escuta, pois, quando se oferta um pouco mais de atenção, é possível notar comportamentos e atitudes que talvez em outra ocasião não viessem perceber. Na maioria das situações a pessoa que necessita de ajuda pode até não dizer, mas está passando por uma série de conflitos, e que em seus pensamentos desorganizados só pensam em tirar a vida como forma de solucionar o problema e acabar com aquela dor enorme que assola o seu interior. O indivíduo pode até não falar, mas o seu "corpo fala", pedindo socorro, atitudes essas reveladas através de mudanças no olhar, através da fala ou comportamentos.

Assim, pode-se concluir que houve um índice de mortes por suicídio relevante no município de Sousa, interior da Paraíba, referente aos anos 2016 e 2017 e que os dados mostram a importância de se trabalhar o assunto, falando das doenças que afligem e que podem ser o gatilho para o desenvolvimento de pensamentos suicidas, não tratando-as como "tabu", como inúmeras vezes nos deparamos com situações desse tipo, negligenciando, talvez até por falta de conhecimento sobre o assunto.

Retratar a necessidade de reconhecer quando o outro está em sofrimento, seja por perdas, atritos interpessoais, dentre outros, de forma que seja possível intervir em tempo precoce, através do reconhecimento de comportamentos e atitudes que estejam assolando pessoas que estão em grau de sofrimento profundo e que necessitam de ajuda para sair desse mar de dores e sofrimento profundo.

Para que seja possível ajudar necessita-se de conhecimento e sabedoria da relevância do assunto e a repercussão na ida do indivíduo que poderá ser causada quanto aos familiares se não tiverem uma visão rápida para os pedidos de ajuda. Só assim, conhecendo o assunto, é possível intervir de maneira correta e precocemente quanto a identificação dos pedidos de socorro.

Dessa forma, espera-se que o estudo possa contribuir de maneira que os profissionais da saúde reconheçam sinais expressos pelos pacientes e consigam 
identificar indicadores de risco, para, assim, intervirem rapidamente, podendo diagnosticar e ajudar mais precocemente na prevenção do suicídio, seja através da orientação, da busca de cuidados especializados para uma avaliação adequada e assim tentar reduzir esses índice de mortes por suicídio.

\section{REFERÊNCIAS BIBLIOGRÁFICAS}

BARRERO S, MATUSEVICH D. Suicídio en la vejez. Buenos Aires: Editorial Polemos, 2009.

BOTEGA, N.J. Prática Psiquiátrica no Hospital Geral: interconsulta e emergência. Porto Alegre: Artmed, 2002.

BRASIL. IBGE. Instituto Brasileiro de Geografia Estatística. 2016. Disponível em: <https://ces.ibge.gov.br/>. Acesso em: 10 de outubro de 2017.

CATALDO NETO, A, GAUER, GJC, FURTADO, NR. Psiquiatria para estudantes de medicina. Porto Alegre: EdiPucrs, 2003.

FIOCRUZ, Portal. Suicídio: pesquisadores comentam relatório da OMS, que apontou altos índices no mundo. 2014. Disponível em: <https://portal.fiocruz.br/pt-br/content/suicidio-brasil-e8o-pais-das-americas-com-maior-indice>. Acesso em: 08 de setembro de 2017.

HALES, R.E.; YUDOFSKY, S.C.; GABBARD, G.O. Tratado de Psiquiatria Clínica. $5^{a}$ Ed. Porto Alegre: Artmed, 2012.

LOPES, H.D. Suicídio-causas, mitos e prevenções. São Paulo: Hagnos, 2007.

LOVISI, G.M. et al. Análise epidemiológica do suicídio no Brasil entre 1980 e 2006 . Rev. Bras. Psiquiatr. São Paulo, v. 31, supl. 2, p. S86-S93, Oct. 2009.

BRASIL, Ministério da Saúde. Conselho Nacional de Saúde. Resolução n. 466, de 12 de dezembro de 2012. Aprova diretrizes e normas regulamentadoras de pesquisas envolvendo seres humanos. Brasília, Diário Oficial da União, 2012. Disponível em: <http://bvsms.saude.gov.br/bvs/saudelegis/cns/2013/res0466_12_12_2012.html>. Acesso em: 16 de outubro de 2017.

LEONEL, Carla. O que leva uma pessoa ao suicídio? 2014. Disponível em: http://medicinna mitoseverdades.com.br/blog-o-que-leva-uma-pessoa-ao-suicídio. Acesso em: 16 de outubro de 2017.

NATRIELLI FILHO, D.G. Suicídio: estudos fundamentais. Rev. Bras. Psiquiatr. São Paulo, 2005 , v. 27 , n. 3 , p. $260-261$.

OMS. Organização Mundial da Saúde. Prevenção do Suicídio: um recurso para conselheiros. Departamento de Saúde Mental e de Abuso de Substâncias. Gestão de Perturbações Mentais e de Doenças do Sistema Nervoso. Genebra: OMS, 2006. Disponível em: $<$ http://www.who.int/mental_health/media/counsellors_portuguese.pdf $>$. Acesso em: 02 de setembro de 2017.

OMS. Prevenção do suicídio :um manual para profissionais da saúde em atenção primária: Genebra, 2000. 
OWENS, D.; HORROCKS, J.; HOUSE, A. Fatal and non-fatal repetition of self-harm: Systematic review. British Journal of Psychiatry, 2002, v.181, p.193-199.

ROTHES, I. Suicídio Juvenil. Representações sociais dos médicos e dos psicólogos. Dissertação de Mestrado apresentada à Faculdade de Psicologia e Ciências da Educação. Porto: Universidade do Porto Sampaio, 2006.

SADOCK, B.J.; SADOCK, V.A. Compêndio de Psiquiatria: Ciências do comportamento e psiquiatria clínica. 9ª Ed. Porto Alegre: Artmed, 2007.

STUART, G. et al. Suicídio e eutanásia. São Paulo, SP: Editora Cultura Crista, 2004,p.9.

VÄRNIK, P. Suicide in the World. International Journal of Environmental Research and Public Health. 2012, v.9, n.3, p.: 760-771.

VARELA, D. Setembro Amarelo: prevenção do suicídio. Rádio Capinzal. 2016. Disponível em: <http://www.radiocapinzal.com.br/noticias/setembro-amarelo-prevencao-do-suicidio/3118>. Acesso em: 18 de novembro de 2017.

VIDAL, C.E.L; GONTIJO, E.C.D.M.; LIMA, L.A. Tentativas de suicídio:Atores prognósticos e estimativa do excesso de mortalidade. Cadernos de saúde pública, 2013, v.29, p.175-187.

WERLANG, B.S.G.; BORGES, V.R.; FENSTERSEIFER, L. Fatores de risco ou proteção para a presença de ideação suicida na adolescência. Revista Interamericana de Psicologia, 2005, v.39, n.2, p.259-266.

WHO. World Health Organization. Country reports and charts available. 2014. Disponível em: <www.who.int/mental_health/prevention/suicide/country-reports/en/index.htlm.>. Acesso em: 27 de setembro de 2017. 